\title{
A Relation Between Binomial Coefficients and Fibonacci Numbers to the Higher Power
}

\author{
Yuhong Che \\ Weinan Normal University, Weinan Shaanxi, 714000, China
}

Key words: Binomial coefficient, Fibonacci numbers, Convolution.

Abstract. In this paper, we calculatate high power of Fibonacci numbers by elementary mathematical methods and prove an interesting identity between the binomial coefficients and the high power of Fibonacci numbers.

\section{Introduction}

Let us begin by recalling the binomial theorem $(a+b)^{n}=\sum_{i=0}^{n}\left(\begin{array}{l}n \\ i\end{array}\right) a^{n-i} b^{i}$,for arbitrary integers $n$ and $i$ with $n \geq i \geq 0$. The coefficient $\left(\begin{array}{l}n \\ i\end{array}\right)$ are known as binomial coefficient and is given by

$$
\left(\begin{array}{l}
n \\
i
\end{array}\right)=\frac{n !}{i !(n-i) !} \text {. }
$$

It is clear from the above formular (1) that $\left(\begin{array}{l}n \\ i\end{array}\right)$ must be a positive integer.The binomial theorem,for arbitrary complex number $\mathrm{x}$ is given by $(1+x)^{n}=\left(\begin{array}{l}n \\ i\end{array}\right) x^{i}$.

Let $F_{l}$ (for abitrary non-negative ${ }^{l}$ ) be the $l-$ th Fibonacci number given by

$$
F_{l}=\frac{\alpha^{l}-\beta^{l}}{\alpha-\beta}
$$

and $L_{l}$ be the ${ }^{l-t h}$ Lucas number defined by

$$
L_{l}=\alpha^{n}+\beta^{n} .
$$

For a long time,properties of binomial coefficients and Fibonacci numbers had been very interesting topics in 'combinatorics' and in 'number', and the identities relating binomial coefficients and Fibonacci numbers attracted many experts.We refer the reader to [1],[2],[3],[4],[5],[6] and [7] for more exhaustive details.

For abitrary fixed nonnegative integer ${ }^{k}$ and abitrary fixed positive integer ${ }^{n}$, we denote the convolution of sequence $\left\{\left(\begin{array}{l}n \\ i\end{array}\right)\right\}_{i=0}^{n}$ and $\left\{F_{k+i}^{m}\right\}_{i=0}^{n}$ by $f(k, m, n)$ and define that as follows:

$$
f(k, m, n)=\left(\begin{array}{l}
n \\
0
\end{array}\right) F_{k}^{m}+\left(\begin{array}{l}
n \\
1
\end{array}\right) F_{k+1}^{m}+\ldots+\left(\begin{array}{l}
n \\
n
\end{array}\right) F_{k+n}^{m}=\sum_{i=0}^{n}\left(\begin{array}{l}
n \\
i
\end{array}\right) F_{k+i}^{m}
$$

The question of calculating $f(k, m, n)$ is being considered in [8],[9],[11],[12] and in [13].For example when $m=1,2,3$, it had been proved that:

$$
f(k, 1, n)=F_{k+2 n}
$$




$$
\begin{gathered}
f(k, 2, n)= \begin{cases}5^{\frac{n}{2}} L_{2 k+n}, 2 \mid n \\
5^{\frac{n-1}{2}} F_{2 k+n}, 2+n\end{cases} \\
f(k, 3, n)= \begin{cases}\frac{1}{5}\left(2^{n} F_{3 k+2 n}-(-1)^{k+n} 3 F_{k-n}\right) & k \geq n \\
\frac{1}{5}\left(2^{n} F_{3 k+2 n}+3 F_{n-k}\right) & k<n .\end{cases}
\end{gathered}
$$

Here $F_{k+2 n}, F_{2 k+n}, F_{3 k+2 n}, F_{k-n}$ denotes the $(k+2 n)-t h,(2 k+n)-t h,(3 k+2 n)-t h$ and $(k-n)-t h$ Fibonacci numbers respectively.Also, ${ }_{2 k+n}$ denotes the $(2 k+n)-t h$ Lucas numbers. Inspired by the above work,a natural question would be whether there exist a similar conclusion to the higher powers of the Fibonacci numbers as well.We try to explore this here in this work.To this end,let $f(k, 4, n)$ be the convolution of sequence $\left\{\left(\begin{array}{l}n \\ i\end{array}\right)\right\}_{i=0}^{i=n}$ and $\left\{F_{k+i}^{4}\right\}_{i=0}^{n}$, i.e.

$$
f(k, 4, n)=\left(\begin{array}{l}
n \\
0
\end{array}\right) F_{k}^{4}+\left(\begin{array}{l}
n \\
1
\end{array}\right) F_{k+1}^{4}+\ldots+\left(\begin{array}{l}
n \\
n
\end{array}\right) F_{k+n}^{4}=\sum_{i=0}^{i=n} F_{k+i}^{4} .
$$

In this paper,we consider this question and prove an identity between the binomial coefficients and the quartic of Fibonacci numbers. In other words,we prove the following result.

Theorem For abitrary fixed nonnegative integer $k$ and positive integer $n$, let $f(k, 4, n)$ be the convolution of sequence $\left\{\left(\begin{array}{l}n \\ i\end{array}\right)\right\}_{i=0}^{n}$ and $\left\{F_{k+i}^{4}\right\}_{i=0}^{n}$, then

$$
f(k, 4, n)=\frac{1}{25}\left[3^{n} L_{4 k+2 n}-4(-1)^{(k+i)} \sqrt{5}^{n+1} F_{2 k+n}+6\right] .
$$

\section{Proof of the theorem}

Proof.It is known that,

$$
F_{l}=\frac{\alpha^{l}-\beta^{l}}{\alpha-\beta},
$$

where

$$
\alpha=\frac{1+\sqrt{5}}{2}, \beta=\frac{1-\sqrt{5}}{2},
$$

Now using (5)、 (6) we get

$$
\begin{gathered}
F_{l}^{4}=\frac{1}{25}\left(\alpha^{4 l}-4 \alpha^{3 l} \beta^{l}+6 \alpha^{2 l} \beta^{2 l}-4 \alpha^{l} \beta^{3 l}+\beta^{4 l}\right) \\
=\frac{1}{25}\left(\alpha^{4 l}-4(-1)^{l} \alpha^{2 l}+6(-1)^{2 l}-4 \alpha^{l} \beta^{3 l}+\beta^{4 l}\right)
\end{gathered}
$$

According (2) and (7) we see that

$$
f(k, 4, n)=\sum_{i=0}^{n}\left(\begin{array}{l}
n \\
i
\end{array}\right) F_{k+i}^{4}=\frac{1}{25} \sum_{i=0}^{n}\left(\begin{array}{l}
n \\
i
\end{array}\right) X
$$


where

$X==\alpha^{4(k+i)}-4(-1)^{(k+i)} \alpha^{2(k+i)}+6(-1)^{2(k+i)}-4(-1)^{(k+i)} \beta^{2(k+i)}+\beta^{4(k+i)}$

We know from (2) that

$$
\sum_{i=0}^{n}\left(\begin{array}{l}
n \\
i
\end{array}\right) \alpha^{4 i}=\left(1+\alpha^{4}\right)^{n}
$$

and

$$
\sum_{i=0}^{n}\left(\begin{array}{l}
n \\
i
\end{array}\right) \beta^{4 i}=\left(1+\beta^{4}\right)^{n}
$$

Now if we use the relations (6) and (7) we find that

$$
1-\alpha=\beta, 1-\beta=\alpha, \alpha \beta=-1,1+\alpha^{4}=3 \alpha^{2}, 1+\beta^{4}=3 \beta^{2}, 1+\alpha^{2}=\sqrt{5} \alpha
$$

Substituting these into (11) we have,

$$
\begin{aligned}
& f(k, 4, n)=\frac{1}{25} \sum_{i=0}^{n}\left(\begin{array}{l}
n \\
i
\end{array}\right)\left(\alpha^{4 k+4 i}-4(-1)^{(k+i)} \alpha^{2 k+2 i}+6(-1)^{2 k+2 i}-4(-1)^{k+i} \beta^{2 k+2 i}+\beta^{4 k+4 i)}\right) \\
& =\frac{1}{25}\left[\alpha^{4 k} \sum_{i=0}^{n}\left(\begin{array}{l}
n \\
i
\end{array}\right) \alpha^{4 i}-4(-1)^{(k+i)} \alpha^{2 k} \sum_{i=0}^{n}\left(\begin{array}{l}
n \\
i
\end{array}\right) \alpha^{2 i}+6(-1)^{2 k+2 i}\right. \\
& \left.-4(-1)^{k+i} \beta^{2 k} \sum_{i=0}^{n}\left(\begin{array}{l}
n \\
i
\end{array}\right) \beta^{2 i}+\beta^{4 k} \sum_{i=0}^{n}\left(\begin{array}{l}
n \\
i
\end{array}\right) \beta^{4 i}\right] \\
& =\frac{1}{25}\left[\alpha^{4 k}\left(1+\alpha^{4}\right)^{n}-4(-1)^{(k+i)} \alpha^{2 k}\left(1+\alpha^{2}\right)^{n}+6(-1)^{2 k+2 i}-4(-1)^{k+i} \beta^{2 k}\left(1+\beta^{2}\right)^{n}+\beta^{4 k}\left(1+\beta^{4}\right)^{n}\right] \\
& =\frac{1}{25}\left[\alpha^{4 k}\left(3 \alpha^{2}\right)^{n}-4(-1)^{(k+i)} \alpha^{2 k}\left(1+\alpha^{2}\right)^{n}+6(-1)^{2 k+2 i}-4(-1)^{k+i} \beta^{2 k}\left(1+\beta^{2}\right)^{n}+\beta^{4 k}\left(3 \beta^{2}\right)^{n}\right] \\
& =\frac{1}{25}\left[3^{n} \alpha^{4 k+2 n}-4(-1)^{k+i} \alpha^{2 k}\left(1+\alpha^{2}\right)^{n}+6-4(-1)^{k+i} \beta^{2 k}\left(1+\beta^{2}\right)^{n}+3^{n} \beta^{4 k+2 n}\right] \\
& =\frac{1}{25}\left[3^{n}\left(\alpha^{4 k+2 n}+\beta^{4 k+2 n}\right)-4(-1)^{k+i}\left\{5^{\frac{n}{2}}\left(\alpha^{2 k+n}-\beta^{2 k+n}\right)\right]+6\right\}
\end{aligned}
$$

Thus finally we have

$$
f(k, 4, n)=\frac{1}{25}\left[3^{n} L_{4 k+2 n}-4(-1)^{(k+i)} \sqrt{5}^{n+1} F_{2 k+n}+6\right] .
$$

The question of calculating $f(k, m, n)$ for $m=5$ or for even higher powers could be solved in the same way but the form may be too complicated.

\section{Acknowledgments}

This research was supported by the Education Department of Shaanxi Province (Grant NO. 16JK1265). 


\section{References}

[1] W. Andrzez,Some identities for the generalized Fibonacci numbers and the generalized Lucas numbers, Applied Mathematics and Computation,219(2013),5564--5568.

[2] J J Chao, The relation between generalized Yang Hui triangle and Fibonacci sequence, Journal of Capital Normal University:Natural Science Edition,32(2) (2011),11--13.

[3] X F Chen,Another relation between Fibonacci number and Yang Hui triangle, Journal of Capital Normal University:Natural Science Edition, 35(3) (2014),1--2.

[4] L K Hua, Begin from Yang Hui triangle[M].Beijing, China Youth Press,1962.

[5] Y Hai, L Bo, and X Gao, A relation between binomial coefficients and cubes of Fibonacci numbers, Basic Sciences Journal of Textile Universities, 28(4) (2015),395-397.

[6] D H MICHAEL, An identity involving binomial coefficients, The Mathematical Gazette, 89 (2005),256--258.

[7] V J W Guo, J Y Liu, and Y Liu, A q-analogue of Zhang's binomial coefficient identities, Discrete Mathematics, 309(20) (2009),5913-5919.

[8] $\mathrm{Y} \mathrm{G} \mathrm{Wu}$, Identities involving powers and inverse of binomial coefficients, Journal of Mathematical Research Exposition,31(6)(2011),1021-1029.

[9] G Z Tu, The method of enumerative combinatory and its application, Beijing: Science press, 1981.

[10]L Z Xu, S M Jiang, and Z Q Zhu .Computational and combinatorial mathematics. Shanghai, Science and Technology Press,1983.

[11]W P Zhang, Some identities involving the Fibonacci numbers, The Fibonacci Quarterly, 35(1997), 225--229.

[12]W P Zhang, Some identities involving the Fibonacci numbers and Lucas numbers, The Fibonacci Quarterly, 42(2004), 149--154.

[13]W P Zhang, Several identities involving the Fibonacci numbers and Lucas numbers. The Fibonacci Quarterly ,45(2007), 164--171. 